\title{
LA INTEGRACIÓN REGIONAL Y LA GÉNESIS DE LA COMUNIDAD POLITTICA EN CENTROAMÉRICA. 1902-1906
}

\section{CENTRAL AMERICA INTEGRATION AND THE GENESIS OF THE POLITICAL COMMUNITY. 1902-1906}

\author{
Edgar Solano Muñoz*
}

\begin{abstract}
RESUMEN
El artículo analiza la formación de la comunidad política regional centroamericana en los inicios del siglo XX. Se enfatiza en el estudio del proceso de integración política centroamericana a partir del Tratado de Corinto de 1902 y la posterior suscripción del Tratado de Paz, Amistad y Comercio de 1906, suscrito en San José, Costa Rica. El concepto central del artículo es el de comunidad política, desde el cual se aborda la dinámica socio-política regional centroamericana y su relación con los procesos de integración regional.
\end{abstract}

PALABRAS CLAVES: COMUNIDAD POLÍTICA * INTEGRACIÓN REGIONAL * HISTORIA POLÍTICA * TRATADOS LIMÍTROFES * PANAMERICANISMO

ABSTRACT

The article analyzes the formation of the Central American political regional community in the beginnings of the XX century. It is emphasized in the study of the process of Central American political integration starting from the Treaty of Corinth of 1902 and the later subscription of the Treaty of Peace, Friendship and Trade of 1906, undersigned in San José, Costa Rica. The central concept of the article is that of political community, from which is approached the Central American regional sociopolitical dynamics and its relationship with the processes of regional integration.

KEYWORDS: POLITICAL COMMUNITY * REGIONAL INTEGRATION * POLITICAL HISTORY * BORDERING TREATIES * PAN AMERICANISM 


\section{INTRODUCCIÓN}

En el siguiente artículo se analiza la gestación de Centroamérica como una comunidad política en los albores del siglo Xx. Este abordaje se hace desde la óptica de los estudios sobre integración regional, específicamente los relacionados con la integración política. Nuestra región ha sido protagonista de múltiples iniciativas integracionistas, al calor del grito de independencia en 1821 surgió la Federación Centroamericana cuya vida se alargó hasta 1842 . Otras tentativas integracionistas encabezadas por caudillos como Morazán, Barrios y Zelaya surgieron después, pero con limitados alcances.

Con la formación de los Estados nacionales en Centroamérica, sobrevinieron las primeras disputas limítrofes. La definición del espacio geográfico de cada estado se hizo considerando las antiguas demarcaciones coloniales, pero dicho criterio se debilitó en la época republicana ante las ambiciones de los grupos dirigentes de cada país por hacerse de más tierra y recursos naturales. Como consecuencia emergieron disputas y conflictos armados que en no pocas ocasiones enfrentaron a las noveles repúblicas de la región.

En el contexto internacional, la región centroamericana pasó a incorporarse dentro de los intereses geo-estratégicos de los Estados Unidos, habida cuenta del interés de la nación del norte por la construcción de un canal interoceánico. Para ello, articuló su propio discurso integracionista, "el Panamericanismo". Mediante dicho discurso, los Estados Unidos establecieron los parámetros para regir sus relaciones (políticas, económicas, sociales, culturales) con América Latina. Las conferencias panamericanas se iniciaron en el último tercio del siglo XIX, y servían de marco regulatorio en la definición de las prioridades norteamericanas en toda la Cuenca del Caribe. Esto tuvo un impacto directo en los procesos de integración, debido a que Estados Unidos privilegió la negociación con grupos de países (ahora bajo la categoría de regiones) antes que con países de forma aislada.

En el caso centroamericano la incidencia de los Estados Unidos en los procesos de integración política empieza en 1906. En este año, se celebró en San José, Costa Rica la primera Conferencia de Paz, Amistad y Comercio regional. En dicha conferencia, se articuló por primera vez en el siglo XX un proyecto de integración regional bajo los criterios de arbitraje obligatorio, resolución pacífica de disputas limítrofes $y$ libre comercio. Aunque condicionada por los factores mencionados con anterioridad, la comunidad política centroamericana se plasma como una "puesta en común" que hacen los Estados de la región en pos de un conjunto de objetivos comunes: paz, fronteras pacíficas, educación y comercio.

Este texto está compuesto por cuatro acápites. En el primero se analiza el contexto histórico en el cuál surge la comunidad política centroamericana, en el segundo se abordan los elementos teóricos que definen a la comunidad política, en el tercero se estudian los conflictos limítrofes de la región y su incidencia en el proceso de integración regional y finalmente, se aborda el tema de Centroamérica y el corolario de la Doctrina Monroe.

\section{LA GESTACIÓN DE LA COMUNIDAD POLÍTICA REGIONAL EN LOS ALBORES DEL SIGLO XX}

El proceso de constitución de los Estados en Centroamérica se acentuó en la segunda parte del siglo XIX mediante la fundación de las repúblicas. Entre 1843 y 1859 uno a uno, los cinco estados centroamericanos se proclamaron ante la comunidad internacional como Repúblicas independientes y soberanas. Así por ejemplo, Guatemala en 1847, Costa Rica en 1848, Nicaragua en 1854, El Salvador en 1859 y Honduras en 1865.

Este paso en la consolidación del Estado Nacional, fue aparejado con la centralización del poder político, la secularización del Estado, la constitución de mecanismos de control y reconocimiento ciudadano $y$, sobre todo la delimitación de sus respectivos territorios. Dicho proceso, finalmente llevará a la construcción de las identidades nacionales mediante la elaboración de discursos e iconografías relacionadas con la nacionalidad, muy propias del último tercio del siglo XIX e inicio del XX (Taracena/Piel, 1995: cap.5). 
En cada una de las repúblicas de la región jugó un papel de primer orden la delimitación de las fronteras. El control efectivo del territorio nacional suponía no solamente la integración nacional de los grupos étnicos y sociales, sino también la delimitación del perímetro geográfico dentro del cual se iban a desarrollar las condiciones del desarrollo material de las nuevas repúblicas.

Para lograr lo anterior se debe contar con un mínimo de condiciones de carácter administrativo estatal que puedan ser subordinadas a dicho gobierno central, fundamentalmente la posibilidad de poder centralizar un sistema de finanzas para la totalidad del territorio (Pinto, 1983:115).

Las implicaciones derivadas de la consolidación territorial de los Estados/Nacionales fueron la base de los conflictos entre los gobiernos del área. La frontera común entre Guatemala y El Salvador fue motivo de constantes pugnas desde fines del siglo XIX, lo mismo entre Guatemala y Honduras, $y$ ni qué decir, de los constantes desacuerdos limítrofes de Costa Rica tanto con Panamá así como con Nicaragua. Estos desacuerdos, en el corto plazo trajeron tensión y desequilibrio regional, pero en el mediano plazo originaron la búsqueda de soluciones mediante la normativa internacional.

El 29 de julio de 1899 se firma la Convención de la Haya, mediante la cual se abrió el espacio en el orden internacional para la resolución de conflictos entre los Estados. Al mismo tiempo, se creó la Corte Permanente de Arbitraje, que iba a funcionar como una instancia mediadora en los conflictos internacionales. Esta convención se ratificó en Centroamérica en 1902, y originó la Convención de Paz y Arbitraje Obligatorio suscrita en el puerto nicaragüense de Corinto, el 20 de enero de 1902, y el Manifiesto de los Centroamericanos en el mismo puerto, el 20 de agosto de 1904. Estos dispositivos jurídicos se constituyeron en el marco resolutorio de los conflictos regionales prácticamente hasta 1948 cuando se firma la carta constitutiva de las Naciones Unidas.

En su contenido, la Convención de
Corinto se amparó en el artículo 26 de la Convención de la Haya, es decir, aquel relacionado con el arbitraje obligatorio para las partes contratantes en caso de conflicto originado por asuntos limítrofes, así como en caso de reclamaciones por daños y perjuicios pecuniarios. Como parte de los procedimientos establecidos por el documento suscrito en la Haya, se adoptó la figura del mediador, el cual podía ser ejercido por el jefe de gobierno de cualquier país parte de la convención que recibiera el beneplácito de los países implicados en un pugilato.

Entre 1902 y 1905, al amparo de la Convención de la Haya, los gobiernos del área firmaron una buena cantidad de convenciones relacionadas con variedad de temas, entre los que tenemos:

$\diamond$ Patentes de invención, dibujos y modelos industriales, marcas de comercio y de fábrica.

$\diamond$ Extradición y protección contra el anarquismo.

$\diamond$ Reclamaciones por daños y perjuicios, asî como arbitraje obligatorio.

$\diamond$ Ejercicio de profesiones liberales, derechos de extranjería, congreso aduanero.

$\diamond$ Ferrocarril panamericano.

$\diamond$ Policía sanitaria.

$\diamond$ Banco panamericano ${ }^{1}$.

Al margen de la variedad y alcances de los acuerdos suscritos por los Estados de Centroamérica a raíz de la Convención de la Haya, lo cierto es que podemos constatar que surge una nueva modalidad de relacionarse entre ellos. En primer lugar, prevalece la idea de que existe un marco jurídico dentro del cual se desarrollan las relaciones entre estados en caso de conflicto y en segundo, que dentro de la región empieza a resurgir la necesidad de presentarse frente al resto del mundo como un solo bloque. Esta postura se adoptó en la Asamblea Iberoamericana celebrada en París en 1905, y luego, en el Congreso Panamericano de Río de Janeiro, del mismo año.

ANCR. Serie Relaciones Exteriores. Caja nro.171. s.f. La abreviatura inicial significa Archivo Nacional de Costa Rica (así se empleará en lo sucesivo). 
La Asamblea Iberoamericana, congregó buena parte de los países hispano-parlantes que habían sido colonias de España, más algunos otros de habla francófona, con el fin de propiciar el debate sobre la expansión del imperialismo sobre todo en Europa, pero con repercusiones en otras partes del orbe. Autodefinidos como "reunión de espíritus emancipados", los países participantes acariciaron la idea de fundar una Federación Ibero-Americana cuyo eje de sus preocupaciones, fuera el respeto de las etnias y la constitución de bloques de países como una forma de resistencia a las tendencias imperiales imperantes en Europa.

Con la participación en este evento, se dio inicio formalmente a una de las tendencias integracionistas más fuertes en Centroamérica y que perduró, incluso hasta los años setenta del siglo XX. El Unionismo Centroamericano se conectaba así con las tendencias integradoras de Europa. Su discurso era claro,

No hay salvación sino en la acción: una acción pronta, eficaz y coordinada hacia la unión (...) grandes por la unión, fuertes por la fraternidad, son esas dos bases de nuestra fuerza étnica y de nuestra grandeza política ${ }^{2}$.

En nuestra región, el Unionismo se personificó en Salvador Mendieta, quien fundó el Partido Unionista Centroamericano (PUCA) el 18 de junio de 1899 en la ciudad de Guatemala. Cinco años después, se organiza formalmente como entidad política en Diriamba Nicaragua, desde donde integra su comité político e inicia los trabajos tendientes para la realización de su primera convención que será celebrada hasta el año de 1912. En años posteriores, el Sr. Mendieta al evaluar en un diario centroamericano los alcances de su movimiento integracionista señaló:

En Nicaragua a pesar del régimen contrario el Unionismo, vibra, trabaja, hace acto de presencia donde quiera que

ANCR. Serie Relaciones Exteriores. Caja nro.164. 1905. s.f. puede... Honduras fue aplastado por larga dictadura, $y$ apenas comienza a balbucear... en Costa Rica aumentan los antes escasos unionistas, pero carecen de coordinación... en Quezaltenango y Los Altos la inquietud unionista es perenne y en esta ciudad de Guatemala, disminuye la onda fría unionista que hallé en 1949, en El Salvador el número de unionistas es enorme, pero disperso ${ }^{3}$.

El segundo momento que consideramos premonitorio con relación a la gestación de proyectos integracionistas en la región, lo encontramos en la celebración del Congreso Panamericano en Río de Janeiro el 11 de diciembre de 1905. A diferencia de la actividad celebrada en París, en esta ocasión la injerencia de los Estados Unidos era evidente. La actividad se enmarcaba dentro de las acciones generadas desde Washington para asegurar su perímetro de influencia política en Latinoamérica, no en vano, dichas conferencias son el preámbulo de la formación de la Organización de los Estados Americanos.

A pesar de lo anterior, en esta ocasión, Centroamérica se presentó como una sola unidad, aún y cuando no existía en la región una sola entidad encargada de la integración regional.

Actualmente nos encontramos divididos en cinco Estados independientes con Panamá como un sexto, los cuales acaso puedan unirse en una nación mayor. Pero unidos o separados los estados de Centroamérica, han demostrado todo el tiempo su amor al progreso y al adelanto, ellos han cooperado con ideas de verdadero panamericanismo ${ }^{4}$.

Los ejes temáticos de la Conferencia Panamericana eran los mismos que inquietaban a los sectores dirigentes de la región, y

El Unionista. El Salvador. 18 de junio de 1972.

"El Congreso Panamericano". Tomado del Jornal do Commercio de Río de Janeiro, 11 de diciembre de 1905. 
sobre los cuales se habían elaborado algunos acuerdos, sobre todo a partir de la Carta de Corinto. Entre ellos se incluyeron los temas del arbitraje, las comunicaciones fluviales, marítimas $y$ terrestres, el tribunal de justicia panamericano, tratados en materia de legislación civil, comercial, penal, marcas, patentes y de ejercicio de profesiones liberales, libre navegación en los ríos, el ferrocarril y la carretera panamericana, el banco panamericano y tratados de comercio.

Es evidente entonces, que los años que dan fin al siglo XIX y la primera década del siglo $\mathrm{xx}$, atestiguan las primeras inquietudes de generar proyectos de integración regional centroamericana. Ya sea desde la óptica de una agrupación política regional como el PUCA, de Salvador Mendieta o desde el ángulo de una potencia del exterior. Unionismo y Panamericanismo, son entonces los dos puntos ideológicos de referencia desde los cuales la región dará sus primeros pasos en el ámbito de la integración política (luego aparecerán otros actores e intereses) y en consecuencia, en la edificación de una comunidad política regional. Sin embargo, el parto iba a ser doloroso, debido a que primero debían ventilarse serias disputas fronterizas entre nuestros Estados.

\section{LOS ELEMENTOS CONCEPTUALES DE LA COMUNIDAD POLÍTICA CENTROAMERICANA}

El estudio de la integración política centroamericana basado en la noción de comunidad política, tiene la peculiaridad de que posee una génesis compleja. Por ello, se nos hace prudente realizar una reflexión sobre tres elementos de tipo conceptual que nos sirven para explicar su gestación en la primera década del siglo xx. En primer término, tenemos la presencia de los Estados Unidos en la región a través de la óptica geopolítica del panamericanismo. Mediante esta visión de la política exterior, la potencia del norte consideró esta zona dentro de sus intereses geo-estratégicos, y por lo tanto sujeta a su injerencia política y militar constante. Basta recordar, que los Estados Unidos a inicios del siglo xx, ya habían llevado a cabo la guerra contra España por la posesión de Cuba (1898), acuerparon la independencia de Panamá (1903), proclamaron el corolario a la doctrina Monroe (1904), y ya se habían apoderado mediante las armas —desde 1848- de California, Nevada, Utah, Colorado, Arizona y Nuevo México. Así, su presencia es una constante que acompaña $y$ condiciona la formulación de proyectos integracionistas centroamericanos durante todo el siglo XX.

En segundo término, se considera de mucha importancia el uso de la noción de comunidad política, debido a que mediante ella, se nos hace posible explicar como los Estados y dentro de ellos, las organizaciones, partidos políticos, movimientos políticos y otro tipo de formaciones, se vinculan de manera horizontal y vertical con otras similares de los países vecinos dentro de un proyecto integracionista. El resultado de dicho conjunto de interacciones es variado, no todas las acciones emprendidas en este campo llegan a buen puerto, de hecho, buena parte de estas iniciativas quedan encalladas en el plano discursivo (Pakkasvirta, 1997: 168).

Finalmente, el concepto de comunidad política se ve complementado con la noción de integración regional. Se considera que Centroamérica presenta en diversos momentos de su historia, una forma de integración que es a la vez, geográfica, funcional y social (Galtung, 1969: 130). La proximidad o vecindad de los territorios facilita la primera cualidad. Con relación a los dos restantes, se puede señalar que entre los países centroamericanos existe una marcada interacción que se plasma en relaciones concretas (políticas, migratorias, comerciales, históricas). Estas cualidades le dan ante otras regiones una especificidad que la diferencian y que a la vez la determinan.

En lo sucesivo la integración política regional, se va a entender como "una cesión de poder", que cada Estado, en igualdad de condiciones se compromete a hacer, con la finalidad de constituir una entidad supranacional (no un gobierno regional) que represente los intereses de todos los estados miembros ante la comunidad internacional (Bela Balassa, 1980). En este mismo sentido, Johan Galtung explica dicha situación de la siguiente manera: "las organizaciones entretejen lazos de interdependencia $y$ las asociaciones vínculos de identidad, $y$ ambos trascienden los clásicos Estado-Nación” (1969: 48). 
Un elemento determinante en el tratamiento teórico de la integración política de la región, es el relacionado con la integración de hecho $y$ la constitución de entidades supranacionales. Desde nuestro punto de vista, estas son definidas por los vínculos que se establecen entre los pueblos centroamericanos, y que van más allá del formalismo puesto de manifiesto en las declaraciones presidenciales o en la fundación de instituciones integracionistas. De acuerdo con Robert Keohane, al interior de las regiones integradas funcionan una serie de principios, normas, reglas y procedimientos para la toma de decisiones que facilitan la reciprocidad intensa en áreas de interés común (1984: Caps. 6-7).

Existe entre los centroamericanos una serie de variables históricas que empleadas en función de la construcción de un esquema de integración, siempre salen a flote. El peso de un pasado colonial común, las primeras experiencias de organización política estatal, la injerencia en lo nacional por parte de Inglaterra y luego de Estados Unidos, la guerra, la paz y el retorno al orden democrático, son solo algunas de las partes integrantes de la herencia política regional. Todas ellas nos permiten hablar de que tanto a lo largo del siglo XIX como del XX, se ha dado la búsqueda permanente de un modelo de integración regional, un modelo que responda a los requerimientos de los puntos álgidos en la historia política regional.

Más que una interpretación de acciones puramente protocolarias, la definición de integración política nos remite a aquella situación en la cual se conforma una "asociación de Estados, deliberada y consciente, tendiente a cambiar la realidad política de la región de acuerdo con determinados fines $y$ en un contexto histórico particular" (Astori, 1994: 65).

\section{LOS PRIMEROS OBSTÁCULOS DE LA COMUNIDAD POLÍTICA CENTROAMERICANA: EL CASO DE LAS DISPUTAS LIMÍTROFES}

Tal y como se mencionó con anterioridad, la constitución del primer esquema de integración política regional del siglo Xx no estuvo exento de incidentes. Con la independencia de Colombia proclamada en 1810 y la posterior definición de sus límites en 1819, el estado colombiano determinó que estos iban a ser los mismos que poseía dentro de la antigua Capitanía General de Venezuela y el Virreinato de Nueva Granada, otrora, demarcados por la corona de España. En su carta constitucional, se estableció que su territorio "se extiende hasta el escudo de Veragua en el Atlántico y hasta el río Chiriquí en el Pacífico"5.

La disputa generada a raíz de la circunscripción del territorio de la naciente Colombia generó conflicto en primer momento con Costa Rica y después de 1903 con Panamá. Como la demarcación que se hizo en aquel momento, no satisfacía los intereses costarricenses por perder buena parte del Escudo de Veragua y parte de Gandoca, se tuvo que recurrir a un arbitraje internacional. El presidente francés Laubet, sirvió de árbitro en dicho diferendo y como resultado se estableció una demarcación que se inclinó por los intereses panameños. De modo que "si la justicia hubiera prevalecido, nuestro país a lo menos habría recuperado una parte de la bahía de Almirante, todo de la cual tiene título legal, contra la posesión por fuerza, único a favor de Colombia no obstante las protestas de Costa Rica" ${ }^{\text {. }}$

En marzo de 1905 mediante un tratado de límites entre Costa Rica y Panamá, firmado por Leonidas Pacheco y Santiago de la Guardia, se baja un poco el tono de la discusión sobre la demarcación limítrofe entre ambos países, pero con el cambio de administración en nuestro país en 1906, la polémica se revive y el laudo Laubet es sometido a nuevos cuestionamientos. Los problemas posteriores suscitados con el laudo en mención se derivaron de la vaguedad con que se establecían los puntos demarcatorios entre las dos naciones.

La carencia de autoridades en la zona fronteriza producía un vacío de poder que

5 ANCR. Serie Relaciones Exteriores. Caja nro. 163. 1905. Correspondencia del Embajador de Costa Rica Joaquín Bernardo Calvo desde Estados Unidos. s.f.

6 ANCR. Serie Relaciones Exteriores. Caja nro. 163. 1905. s.f. 17 de enero de 1905. José Astúa Aguilar Ministro de Relaciones Exteriores. 
convertía aquellos lugares en tierra de nadie. Así, un alcalde costarricense de la zona colindante a Panamá, dijo que:

Aunque sé perfectamente que desde la boca del río Coto aguas arriba es la línea marcada por el statu quo entre Costa Rica y Panamá, [debo comunicar] que se ha formado en toda esa línea un caserío bastante numeroso en que por las circunstancias especiales... hace que impere la ley del más fuerte o la del más osado: las cuestiones más baladíes son dilucidadas escopeta o cuchillo en mano, quedando la razón de parte del vencedor?

A la inesperada independencia de Panamá en 1903, se sumó otro factor que incidió en el diferendo limítrofe con Costa Rica. Este fue la presencia de compañías bananeras norteamericanas en las cercanías de lo que después se iría a constituir en la zona del canal. La "American Banana Company", ocupaba unas tierras cercanas a Gandoca y al río Changuinola, como era claro, destinadas al cultivo de la fruta. La empresa en cuestión, se valía del diferendo entre los dos países para ocupar las tierras y desconocer los derechos de ambos países debido a que

... en la actualidad entre Costa Rica y Panamá (no hay) ningún arreglo tocante al dominio y administración del mencionado territorio, (Gandoca) mientras la cuestión de fronteras se resuelve definitivamente... [y] mientras el lindero no se haya establecido de modo firme, el gobierno de los Estados Unidos niega que Costa Rica o Panamá tengan facultad de soberanas para ejecutar sentencias relativas a la apropiación de tales tierras por sus ocupantes ${ }^{8}$.

ANCR. Serie Relaciones Exteriores. Caja nro. 170. s.f.

ANCR. Serie Relaciones Exteriores. Caja nro. 163. s.f. Nota enviada por el Ministro de Relaciones Exteriores, José Astúa Aguilar al ministro plenipotenciario de los Estados Unidos, William Lawrence Ferry. 3 de mayo de 1906.
Las gestiones posteriores realizadas por el gobierno de Costa Rica fueron no ante Francia, sino ante los Estados Unidos, en virtud de los intereses de la compañía antes mencionada. De hecho, nuestro gobierno giró instrucciones al embajador costarricense en Washington, Pedro Pérez Zeledón, para hacer la menor cantidad de concesiones ante los norteamericanos.

En caso de convertir una nueva línea fronteriza y el gobierno de Panamá insistiere en obtener ventajas por el lado del Pacífico, estas tendrán que tener necesariamente su justa compensación por el lado del Atlántico, basadas en el laudo arbitral tal y como lo interpreta Costa Rica, esto es salvando siempre para ésta República toda la región de Talamanca, el río Changuinola, que según la correspondencia del ministro Peralta fue a primera línea que el árbitro contempló como frontera... los E.U. pueden estar interesados en obtener concesiones en el Golfo Dulce... y la isla del Coco... [por tanto] puede oír cualquier proposición, la cual deberá ser comunicada de inmediato al gobierno ${ }^{9}$.

Más allá de estas diferencias limítrofes, que incluso no fueron resueltas sino hasta los años sesenta del siglo Xx, el diferendo entre

9

ANCR. Serie Relaciones Exteriores. Caja nro.170.s.f. Instrucciones al embajador Pedro Pérez Zeledón en Washington. p.1.2.3.

El Washington Post, informó sobre el diferendo con la nota que reproducimos a continuación: Calvo and Root Confer

Costa Rican Minister discusses old boundary dispute "M. Calvo the Costa Rican Minister called on secretary root yesterday and discussed with him the boundary dispute between Costa Rica and Panamá, which involves the interests of two American corporations, the United Fruit Company and the American Banana Company. For years the boundary between Colombia and Costa Rica was doubt, and finally and arbitration was arranged. When Panama seceded, Costa Rica and Panama framed a treaty to settle the dispute, which Panama inherited from Colombia. This treaty has not been ratified as yet, and the American corporations are not satisfied with the boundary agreed upon" 26 de noviembre de 1906. 
costarricenses y panameños en el que terciaban los intereses comerciales norteamericanos, nos muestra uno de los elementos que propició el desarrollo de mecanismos regionales para la solución de controversias regionales. Aunque al momento de la controversia entre Panamá y Costa Rica, ya se caminaba en la región hacia la construcción de tales mecanismos, (el Tratado de Corinto data de 1902) lo cierto es que en aquel momento se recurrió a lo que disponía la Carta de la Haya, es decir, al arbitraje. El resultado no fue satisfactorio para ninguna de las dos partes, pero más allá, evidenció la necesidad de horizontalizar las relaciones políticas entre los estados de Centroamérica.

El Tribunal de Arbitrios Centroamericano, surge a raíz de la necesidad sentida por los Estados de la región, por poseer un instrumento que sirviera de plataforma para la negociación en caso de conflicto. Ningún país centroamericano, se libró de disputas con sus vecinos debido al tema del espacio nacional. La necesidad de integrarse para resolver los problemas derivados de las disputas territoriales, así como la búsqueda de la paz entre Estados mediante el diálogo, es el eje de los nacientes proyectos integracionistas centroamericanos surgidos en la primera década del siglo XX.

Tan solo cuatro años después de la suscripción del Pacto de Corinto, surgió la primera ocasión para ponerlo en ejecución. Un conflicto armado entre Guatemala, El Salvador y Honduras dio la posibilidad. La versión oficial sobre las razones del conflicto, se centraron en una disputa entre los tres países debido a rencores y suspicacias por la financiación de la construcción del ferrocarril guatemalteco al Caribe. La conspiración, planeada desde El Salvador por un grupo de cafetaleros alemanes desafectos con el gobierno de Manuel Estrada Cabrera, provocó la incursión dentro del territorio guatemalteco de fuerzas irregulares. Ello, caldeó el ánimo del presidente Cabrera y declaró sin demora, la ruptura de relaciones con sus vecinos del sur y este.

Con relación a este evento, el presidente guatemalteco, indicó en un diario nicaragüense que:

El conjunto de hechos ocurridos en Guatemala en la emergencia recién pasada, forma el acontecimiento histórico más importante después de nuestra independencia, sus consecuencias internacionales son de tal magnitud que la campaña nacional de 1906 abre una era completamente nueva para Guatemala ${ }^{10}$. [Más adelante señaló, que declaraba la guerra en virtud de que] El territorio nacional se encuentra invadido desde el día de ayer por fuerzas considerables de El Salvador, complementando así la hostilidad que ha demostrado por medio de diversas facciones que armó contra Guatemala con el objeto de perturbar el orden y la paz de la República ${ }^{11}$.

La fortaleza de las relaciones entre los Estados centroamericanos no ha sido precisamente su principal virtud al inicio del siglo XX. Las acusaciones de mutuas intrigas, conatos de rebelión de un país hacia otro, sospechas de confabulaciones políticas y usurpaciones territoriales, eran casi cotidianas. Cualquier opinión respecto a la situación política del país vecino sacada de contexto, era excusa para proferir insultos y motivo para velar armas. La falta de confianza era la norma, el respeto era la excepción. Esto quedó de relieve en las palabras del presidente Estrada Cabrera, cuando alegó que:

La hostilidad del gobierno salvadoreño se acentúa más, en el hecho de permitir en Acajutla el desembarque de elementos de guerra que lleva el vapor "Empire State" (proveniente del puerto de Corinto, Nicaragua) indebidamente bajo la bandera americana, $y$ en que trenes expresos sean conducidos a su destino por la línea férrea de Santa Ana ${ }^{12}$.

10 Diario del Comercio. Managua, Nicaragua. 29 de agosto de 1906. p.1.

11 Alcance a: El Guatemalteco, nro. 96, "Declaratoria de Guerra de Manuel Estrada Cabrera". Guatemala, 10 de julio de 1906.

12 ANCR. Serie Relaciones Exteriores. Caja nro. 171 Secretaría de Estado, Guatemala, 26 de junio de 1906. págs. $2,3$. 
Con la ruptura de relaciones entre Guatemala y El Salvador lo consiguiente fue la declaratoria de la guerra. En una minuta redactada por la Secretaría de Estado de Guatemala, se indicó que en el movimiento revolucionario contra el presidente Estrada Cabrera, se organizó invadiendo el país por cuatro puntos con muchas columnas de hombres armados, provistos de los mejores rifles modernos

El General Barillas (un latifundista de esa región guatemalteca) burlando toda la vigilancia cruzó la frontera y penetró en el territorio... cerca de Motozintla, con 200 hombres bien montados $y$ gran cantidad de rifles... en marcha a Quezaltenango... José León Castillo tomó el puerto de Ocós con poca resistencia... y también invadió por Belice, entrando por el Petén, a la cabeza de 2000 hombres... incluyendo tropas regulares salvadoreñas y emigrados políticos guatemaltecos ${ }^{13}$.

La reacción de los gobiernos de Costa Rica y Nicaragua, con relación al conflicto en el norte de Centroamérica, se inclinó por apelar a los postulados del Tratado de Corinto. Sin embargo, ninguno de los Estados implicados en la guerra de 1906, validaron tal posición, por conocerse que el documento no poseía carácter vinculante. En primer lugar, por tratarse del único instrumento jurídico de su naturaleza suscrito en la región, no se tenía claro, si sus postulados eran de acatamiento obligatorio por parte de nuestros Estados y en segundo término, no existía hasta aquel momento, registro de prácticas regulatorias de las relaciones políticas entre los estados centroamericanos, en el marco de un instrumento jurídico de tal naturaleza. Por ello, los gobernantes de Honduras y Guatemala, consideraron que se trataba solo de un acuerdo regional de buena $f^{14}$.

13 Aquí ofrecemos una visión reducida de los eventos, pero todo el detalle de la rebelión se describe con amplitud en la crónica publicada por el Diario El Hogar. México, 30 de mayo de 1906. págs. 2, 3, 4, 5, 6.

14 Una aclaración de Joaquín Bernardo Calvo, sobre los tratados invocando un compendio de jurisprudencia
La reacción del presidente nicaragüense, José Santos Zelaya, mostró dos facetas. Por un lado, no avaló la reflexión desarrollada por Joaquín Bernardo Calvo, con relación al carácter vinculante del tratado, $y$ por otra, rechazó una solicitud de la cancillería hondureña, para que sirviera de mediador entre las partes en conflicto. En virtud de ello, los gobiernos de Costa Rica y Honduras estimaron que dicho comportamiento "invalida en su totalidad esta convención, [Pacto de Corinto] y en consecuencia no puede continuar funcionando el Tribunal de Árbitros establecido por la misma"15.

El vacío creado por la determinación de los gobiernos de Costa Rica y Honduras, respecto al Tribunal de Arbitraje, hizo que el gobierno guatemalteco iniciara gestiones para buscar un mediador externo. La salida fue, apelar a la colaboración de México en la persona de Porfirio Díaz, y en el presidente de los Estados Unidos, Theodore Roosevelt. Para ello, "el presidente de Guatemala Manuel Estrada Cabrera dio al cuerpo diplomático una recepción... [y] dijo que los guatemaltecos tenían que agradecer la intervención arbitral de los presidentes

internacional de 1768, señalaba que: "Los tratados por ser esencialmente contratos de buena fe, deben ante todo interpretarse en el sentido de equidad $y$ del derecho estricto. Es igualmente regla atenerse más bien al espíritu que a la letra de las convenciones y no dar un valor secundario al sentido literal de las palabras, buscando ante todo cuál ha podido ser la intención común de las partes contratantes". pág. 270, 271. Martens, el sabio profesor de San Petersburgo, dice: "al igual de los contratos privados, deben las convenciones internacionales ejecutarse en buena fe; de otro modo, de nada valdrían. La ejecución BONA FIDE supone el respeto, no solo para la letra sino también para el espíritu del tratado; y carece de fundamento la diferencia que hacen algunos autores entre cláusulas principales y cláusulas secundarias. Todos los artículos de un tratado cubiertos con el mismo grado por la firma y ratificación de su poder soberano, son obligatorios al mismo título. El no observar uno de ellos, aún el menos importante equivale a violar el tratado" Droit Internacional, T I. 113.

15 ANCR. Serie Relaciones Exteriores. Caja nro. 169. 1904-1906. Diario Oficial. El Salvador, San Salvador, 13 de febrero de 1907, \#37, p 267, 269, 268. 
Roosevelt y Díaz que asegura la tranquilidad centroamericana de una manera definitiva"16.

En términos del proceso de integración regional, el conflicto entre Guatemala, El Salvador y Honduras de junio de 1906, fue beneficioso. La mediación ofrecida por el gobierno de México y de Estados Unidos resultó en una reunión para negociar la paz a bordo de un buque de guerra de la marina de los Estados Unidos llamado Marblehead. La convocatoria fue hecha "bajo la excitativa de los presidentes Teodoro Roosevelt y Porfirio Díaz, [con la cual] los gobiernos de Guatemala y El Salvador han firmado la paz a bordo del crucero americano "Marblehead" en alta mar"17.

16 Diario del Comercio. Managua, Nicaragua, 15 de agosto de 1906. p.1.

ANCR. Serie Relaciones Exteriores. Caja nro. 169. 21 de julio de 1906. s.f. El mensaje fue enviado por el secretario de Estado en los términos que reproducimos a continuación. Llama la atención que buena parte del texto estaba cifrado de modo que no disponemos de todos los datos de dicha convocatoria.

Convocatoria del Secretario de Estado Ferry. E.U.

"The president sent today the following telegram to the president of the Salvador and mutatis mutants to the president of Guatemala...to take immediate steps towards settling questions pending with Guatemala either by agreement to arbitrate or by direct negotiation for a definitive agreement between the two countries disturbance of the peace of Central America inflicts grievous injury upon the affected states and causes the gravest concert to the United states whose sole desire is to see its neighbors at peace the recent deplorable renew ant of hostilities should not be allowed to be the precursor of a protracted and disastrous struggle perhaps involving other states and leading to results of which the scope cannot be foreseen in the interest of humanity and the indispensable peace of Central America it becomes my duty to urge a settlement before it may be too late, I offer the deck of the American ship of war Marblehead now on the way to the coast of Salvador as a neutral place where representatives of Salvador and Guatemala may meet to considerer terms of the agreement and armistice between the contestants being memorial effected, I am telegraphing in the same sense to the president of Guatemala may action has the full concurrence of the president of Mexico. Theodore Roosevelt". Washington, 13 de julio de 1906. El resto del mensaje está cifrado.
Los acuerdos de Paz de Marblehead, incluyeron no solo el cese al fuego, sino la firma de un compromiso para la celebración de la primera conferencia de paz centroamericana. La sede de dicha conferencia sería en San José, Costa Rica, y se acordó que fuera en conmemoración de la independencia, el 15 de setiembre de 1906. En lo sucesivo, se le conoce como la conferencia de Paz, Amistad y Comercio de San José. La conferencia en cuestión, representa el primer eslabón en la constitución contemporánea de la integración política regional, en ella se determinaron las normas básicas de las relaciones políticas, comerciales y sociales por parte de cuatro de los cinco estados centroamericanos.

El presidente José Santos Zelaya de Nicaragua, se negó a participar en la Conferencia de San José, alegando que dicha conferencia reñía o desestimaba lo estipulado en la Carta de Corinto, firmada cuatro años antes. La agenda se construyó sobre la base de las necesidades inmediatas y de mediano plazo de nuestros estados. Entre ellas se tuvo en consideración:

El Tratado General de Paz, Amistad y Arbitraje obligatorio de Centroamérica, las normas centroamericanas sobre extradición y comercio, la Convención para el establecimiento de la oficina internacional Centro América con sede en Guatemala, la Convención para el establecimiento del Instituto Pedagógico Centroamericano $y$ el fin a los conflictos entre Guatemala, El Salvador $y$ Honduras ${ }^{18}$.

La Conferencia de Paz, Amistad y Comercio Centroamericana, abrió el espacio para la constitución de Centroamérica como una comunidad política. Nunca antes en la historia regional, nuestros estados habían reglamentado sus relaciones políticas, económicas y sociales al nivel que se hizo en aquella ocasión. El representante de Costa Rica, Joaquín Bernardo Calvo al realizar su discurso en la reunión, se refirió a las cualidades de Centroamérica como región indicando: ANCR. Serie Relaciones Exteriores. Caja nro. 171. s.f. 31 de octubre de 1906. 
Una misma es nuestra sangre, unas mismas nuestras costumbres, unos mismos nuestros ideales; $y$ aún nuestras instituciones $y$ leyes escritas con pequeñas variantes, son también unas mismas. Unificar la legislación y aún el régimen político después de que nos unan el interés y un afecto más positivo, factores de mayor importancia que nuestras solas aspiraciones y nuestras solas voluntades ${ }^{19}$.

El evento anterior significó mucho para los procesos de integración en la región centroamericana del siglo XX. En aquella ocasión, por primera vez los gobiernos del área pensaron en un modelo de integración política no federativo, tal y como fue la norma en el siglo XIX. Una vez roto el pacto colonial y ubicándonos en 1824, la región siempre promovió la idea de una integración política utilizando como base, una estructura de poder federal centralizada, conformada por "Estados asociados", sujetos a una capital y a un congreso regional.

La idea anterior, se rompe en 1906 en la conferencia de San José. La utilización de la noción de comunidad política como el eje de los proyectos de integración política, viene a sustituir el viejo concepto federalista, —valga recalcar que para entonces, cada país centroamericano ya se concibe como una República, libre y soberana-y los términos de referencia para la constitución de los diversos esquemas de integración política han de partir en lo sucesivo, de la puesta en común que, Estados constituidos $y$ reconocidos como tales internacionalmente, hacen sobre la base de una "comunidad de intereses y objetivos" (Chantal Mouffe, 1993: 6).

La agenda acordada para la conferencia de Paz en San José, fue amplia. No solo se trató de establecer un marco de respeto mutuo entre los Estados de la región, sino que también se quiso incursionar en temas como la migración, el comercio, la educación y la fundación de una oficina de asuntos centroamericanos. También se dio origen a la fundación de las primeras instituciones de la integración regional. Por

ANCR. Serie Relaciones Exteriores. Caja nro. 169. Libro de cuentas y planillas. 1906. s.f. ello, consideramos que este es el momento en el que va tomando forma la constitución de una comunidad política regional, ya que es a partir de esos objetivos comunes que se van desarrollando iniciativas posteriores de integración regional.

Los años posteriores a la realización de la "Conferencia de San José", las tentativas para desarrollar un esquema de integración política en la región quedaron soslayadas por el surgimiento de regímenes autoritarios. No en vano, al periodo 1930-1960 se le conoce en la historia de Centroamérica, como la "época de las dictaduras", caracterizado por "censuras de prensa, exilio y cárcel para la oposición, extendido control policiaco, burocracia estatal reducida y dócil, asuntos de hacienda y finanzas en manos de connotados miembros de las familias cafetaleras y generoso trato a compañías extranjeras" (Pérez, 1986: 85).

En síntesis, el Tratado de San José planteó una serie de temáticas que van a surgir constantemente en los sucesivos intentos por desarrollar la integración regional. Su herencia fue significativa, el arbitraje obligatorio, el comercio intra-regional, el tema migratorio, la definición de los límites territoriales y marítimos y la fundación de las primeras instituciones de la integración regional. Pero más allá de estos activos para futuras iniciativas integracionistas, lo cierto es que el proceso de paz de 1906 , puso a la región en el orden de los intereses geo-políticos de los Estados Unidos. La mediación del presidente Roosevelt, no fue solo un acto de buena voluntad, sino más bien la incorporación de la región dentro de las directrices del panamericanismo.

\section{CENTROAMÉRICA Y EL COROLARIO DE LA DOCTRINA MONROE}

Como quedó de manifiesto con anterioridad, la participación de los Estados Unidos como mediador en la guerra del norte de Centroamérica en 1906, no fue gratuita. Esta, sirvió de plataforma para que el panamericanismo como ideología del control y dominación política entrara en escena en Centroamérica. Ya con antelación, la Doctrina Monroe y su 
Destino manifiesto (1823), derivaron en la constitución de zonas de influencia norteamericanas a lo largo de toda la Cuenca del Caribe. En Centroamérica, la Doctrina Monroe llegó a su clímax con la firma en 1850 del Tratado Clayton-Bulwer, entre Estados Unidos e Inglaterra. Mediante dicho tratado, se estableció un conjunto de reglas del juego entre ambas potencias, respecto al manejo de sus intereses en Centroamérica, pero específicamente en lo tocante a la construcción de un canal interoceánico en Nicaragua u otra zona de la región. Este acuerdo complementó lo dispuesto tan solo tres años antes mediante el Tratado Mallarino-Bidlack ${ }^{20}$. Todo esto, venía a ser otro paso en la estrategia norteamericana de anexarse territorios $y$ de incidir de manera directa en la política de amplias zonas del continente, "con la anexión de Puerto Rico, Filipinas y Guam y la ocupación de Cuba, queda manifiesta la articulación entre el Pacífico y el Caribe en función a la estrategia de defensa de los Estados Unidos"(Hernández, 1999: 129).

Ya en los albores del siglo Xx, los Estados Unidos habían promulgado su posición respecto a Centroamérica, mediante lo que se denominó "el corolario de la Doctrina Monroe". En un mensaje del presidente Roosevelt al Congreso, en enero de 1906, señaló que:

Lo que este país desea, es ver a los países vecinos bien organizados, legalmente gobernados y prósperos... si se actúa con eficacia $y$ decencia en todas las cuestiones políticas y sociales, si se conserva el orden y cumplen sus obligaciones, no debe temer ningún país, la intervención de parte de los Estados Unidos"21.

En la misma ocasión, añadió el SubSecretario de Estado, Mr. Lummis que:

Si todos los países bañados por el mar Caribe diesen señales de progreso estable

Tratado Clayton-Bulwer. Gobierno de los Estados Unidos. Texto original íntegro, versión en inglés. 19 de abril,1850.

Diario el Comercio. Managua, Nicaragua, 17 de enero de 1906, pág. 3. $y$ de buen gobierno como con la ayuda de la Enmienda Platt ha dado a Cuba, después que nuestras tropas abandonaron la isla... la elección no puede ser más clara ni más terminante y los pueblos de hispano-América en especial los bañados por el Mar Caribe, no deben olvidar que el "big stick" está suspendido sobre sus cabezas $^{22}$.

Durante la primera década del siglo xx la incorporación de Centroamérica dentro de los intereses continentales norteamericanos toma fuerza. Los Estados Unidos tienen plena conciencia de la posición geoestratégica del istmo, tanto como puente hacia América del Sur, así como de cara a los intereses comerciales europeos en el Caribe. El binomio seguridad-comercio, sirve de base en su argumento de dominación. Un diario nicaragüense, registró una nota, mediante la cual, las autoridades del Departamento de Estado indicaban a sus homónimos nicaragüenses que:

... en lo sucesivo tanto en la correspondencia como en los documentos oficiales tanto impresos como manuscritos, usarán la palabra América en vez de Estados Unidos... ise trata de que ante el mundo América sean los Estados Unidos y que no halla más América del Sur, América del Centro, América del Norte, sino una sola América y que esta América única quiera decir Estados Unidos! $!^{23}$.

La reacción de una parte de los sectores políticos nicaragüenses, fue de repudio al contenido de la nota. Para ellos, la misiva ponía sobre el tapete los verdaderos intereses de los Estados Unidos sobre Centroamérica.

Esa sencilla nota en la que se transparenta toda la astucia ladina del coloso del norte es

Diario el Comercio. Managua, Nicaragua, 17 de enero de 1906, pág. 4. Alocución del SubSecretario de Estado, Mr. Lummis al Congreso. marzo de 1905. p.3. 
una mistificación internacional enorme... gracias a esa política, Cuba, Puerto Rico y Filipinas son otros tantos mercados para la industria yanqui, México va perdiendo su nacionalidad, Panamá será una factoría de los Estados Unidos y, ahora parece que con un golpe maestro quiere declarar a todas las repúblicas de este continente como colonias nominales de la Gran República del Norte... mercado, mercado para su plétora de productos que amenazan ahogarla que no la dejan respirar, que necesita colocar en cualquier parte, por cualesquiera medios... allí la síntesis de la política yanqui ${ }^{24}$.

La articulación funcional del Panamericanismo como el eje de la política exterior norteamericana en Centroamérica y el resto de América Latina siguió varios caminos. El de mayor peso, fue la instauración de las conferencias panamericanas, que con el paso del tiempo dieron origen a la formación de la Organización de Estados Americanos (OEA). El Panamericanismo surge de la intención de los Estados Unidos por establecer acuerdos o tratos con conglomerados de Estados bajo el criterio de la regionalización. Es así, que bajo dicha doctrina, se firman múltiples acuerdos de cooperación económica y asistencial de carácter regional, pero teniendo como norte el resguardo de los intereses económicos y políticos de los Estados Unidos. La configuración del sistema panamericano inicia en 1889 cuando se realiza la primera conferencia panamericana en Washington. Tras esta, se promulgaron los principios básicos de la organización: 1. la regulación de las relaciones políticas y jurídicas entre los Estados en aras de la "coexistencia pacífica" y, 2. la definición del marco para la cooperación y las relaciones económicas entre América Latina y los Estados Unidos (Castañeda, 1956: 337).

Con relación a Centroamérica, la agenda propuesta por la Unión Panamericana implicó: programas de asistencia social de vacunación y ataque a las enfermedades tropicales, la construcción de caminos, casas populares, apoyo

24 Diario El Comercio. Managua, Nicaragua 15 de marzo de 1905. p.4. financiero a los gobiernos del área, asistencia militar y sobre todo la construcción de la infraestructura básica para desarrollar actividades comerciales de carácter regional. Por ejemplo, en 1906 se dio a conocer por el Departamento de Estado norteamericano que:

[el] vicepresidente de la compañía del ferrocarril Panamericano va a las repúblicas de Centroamérica a obtener de sus gobiernos la concesión del establecimiento de un ferrocarril que atraviese los cinco países, partiendo de la frontera mexicana, en donde entroncará con el ferrocarril panamericano de México yendo a conectar con Panamá... la línea proyectada tendrá la extensión de 2500 kms, conectará probablemente con otros ferrocarriles de América Central, como la central de Guatemala, el de Acajutla a Santa Ana en el Salvador y el del gobierno de Nicaragua ${ }^{25}$.

Uno de los resultados tangibles de la participación regional dentro de las reuniones panamericanas, fue la fundación en la ciudad de Guatemala, de una Oficina Internacional de Asuntos Centroamericanos. De forma paralela a la Corte Centroamericana de Justicia la Oficina Internacional Centroamericana, se encargó de ser la instancia encargada de representar a la región ante el resto del mundo. El presupuesto para su operación era facilitado por el gobierno de los Estados Unidos, pero con un ligero aporte de los gobiernos del área. Por ejemplo, "para 1907-8 rondaba los $\$ 36000$ y entre 1908-09 los $\$ 54000 " 26$. En cuanto a sus ámbitos de trabajo, la Oficina Internacional Centroamericana, se ocupaba de una agenda estrictamente integracionista. En ella, se aglutinaban temas como: la "reorganización pacífica de Centroamérica", la educación pública uniforme, el desarrollo del comercio, la agricultura y la industria, así como la elaboración de códigos aduanales análogos $y$ hasta incluso, el logro de mayores niveles de salud y salubridad regional.

Diario del Comercio. Managua, Nicaragua. 1 de setiembre de 1906 p.1.

26
ANCR. Serie Relaciones Exteriores. Caja nro.167. 1906. s.f. 
Una muestra interesante de la recepción del panamericanismo en la región centroamericana, quedó plasmada en la III ${ }^{a}$ conferencia Panamericana, realizada en Río de Janeiro en julio de 1906. Aquella ocasión, sirvió para dar a conocer la percepción de los centroamericanos respecto a las posibilidades de integrarse $y$ de obtener beneficios de ello.

Ser pequeños, relativamente no es una desgracia. La grandeza material si es ciertamente deseable, no es el pináculo de la grandeza, y nosotros sabemos que ésta existe donde la justicia impera, donde el bien general es la ley suprema y donde la aspiración se dirige hacia la consideración $y$ respeto de las otras naciones hacia el mejoramiento común de la humanidad. Actualmente nos encontramos divididos en cinco Estados independientes, con Panamá como un sexto, los cuales acaso puedan unirse en una nación mayor. Pero unidos o separados los estados de Centroamérica han demostrado todo el tiempo su amor al progreso y al adelanto ellos han cooperado con ideas de verdadero panamericanismo al éxito de la I y II conferencia ${ }^{27}$.

Las conclusiones de la III ${ }^{\text {a }}$ Conferencia Panamericana reflejan de manera clara, por donde se orientaron los intereses de los Estados Unidos en materia de sus relaciones con el resto de América. La articulación de un mercado interamericano fue el eje de sus preocupaciones. Por ello, se acordó:

... compilar y distribuir datos comerciales, así como la legislación aduanera y clasificar todo lo referente a tratados $y$ convenciones de las repúblicas americanas, informar sobre asuntos de educación, y funcionar como comisión permanente, compuesta por los representantes

27 Jornal do commercio. Río de Janeiro, 11 de diciembre de 1905. "El Congreso Panamericano. La presentación de Centroamérica”.

ANCR. Serie Relaciones Exteriores. Legación de Costa Rica en Washington. Conclusiones III ${ }^{\mathrm{a}}$ Conferencia Panamericana. Río de Janeiro, Brasil, 11 de agosto de 1906. Caja nro. 169. 1906. s.f. diplomáticos de cada país americano destacado en Washington ${ }^{28}$.

Adicionalmente, se incorporó otro conjunto de temas tales como: el arbitraje internacional en caso de conflicto, las comunicaciones fluviales-marítimas y terrestres, el tribunal panamericano, acuerdos en materia de legislación comercial-penal-judicial-marcas-patentes$y$ de profesionales, ferrocarril panamericano, banco panamericano y tratados de comercio. Aunque el Panamericanismo, fungió como la herramienta fundamental de la articulación de la política exterior de los Estados Unidos respecto al continente americano, también se registró algún nivel de resistencia a la propuesta hegemónica venida del norte.

En París, en ocasión de la celebración de una de las primeras cumbres de latinoamericanistas europeas (1905), se puso sobre el tapete de discusión, la incidencia del Panamericanismo en el destino de los Estados americanos. La remembranza al pensamiento de Simón Bolívar, actúa como la matriz doctrinaria que acompañó a la contrapropuesta del Panamericanismo. El objetivo de aquella actividad, se definió como el de aglutinar y reunir a "una asamblea de la raza libre, a la reunión de espíritus emancipados, suceder de los pueblos amenazados... y hacerla nuestra contra el Imperialismo"29. En la cita de París de 1905, también salió a colación otra corriente ideológica integracionista que tuvo presencia por más de 60 años en Centroamérica, el unionismo. Esta corriente, tuvo en el ámbito regional a sus mayores exponentes en Salvador Mendieta, Joaquín Rodas, Marciano Castillo y Aniceto Montero. El unionismo propugnaba por el desarrollo del espíritu unionista centroamericano, mediante la formación de un Partido de corte regional (PUCA), el desarrollo de la ciudadanía, la instrucción de los pueblos y la intervención del Estado como promotor del desarrollo (Silva, 2005: 2).

De forma adicional, la percepción unionista presente en la Asamblea de París, llevaba 1905. s.f. Asamblea Iberoamericana de París, 1905. 
al plano continental los anhelos unionistas. Por ello, en aquella ocasión, se propuso,

... arrojar las bases de una Federación Ibero-Americana, [ya que]... no hay salvación sino en la acción, una acción pronta, eficaz y coordinada hacia la unión. Es necesario oponer a la teoría del Imperialismo, la del Unionismo. Ante la invasión la cohesión. Proclamemos ante el mundo nuestra voluntad de vivir como pueblos libres $y$ afirmemos ante la historia, nuestra resolución de resistir como una raza heroica. Grandes por la unión, fuertes por la fraternidad, son esas dos bases de nuestra fuerza étnica y de nuestra grandeza política ${ }^{30}$.

\section{CONCLUSIÓN}

El surgimiento de la comunidad política centroamericana en la primera década del siglo $\mathrm{XX}$, fue un proceso lleno de saltos y brincos. La firma del Tratado de Corinto en 1902, abrió el camino para que nuestros Estados en calidad de repúblicas libres y soberanas, se incorporaran en una comunidad de intereses, que aquí hemos denominado, comunidad política centroamericana.

Con la suscripción del Tratado de Paz, Amistad y Comercio de 1906, se establecieron los parámetros que sigue el proceso integracionista hasta la conformación de la Organización de Estados Centroamericanos en 1950. De su seno, surgen las instituciones y posturas discursivas que procuran la integración política regional. En lo sucesivo, se va a atestiguar que los procesos integracionistas en la región oscilan entre tres posturas: 1. integración de la comunidad política como una estrategia para el logro de objetivos económicos, 2. la constitución una comunidad política como una forma de expresión de los anhelos y las aspiraciones de los actores socio-políticos regionales en las diversas coyunturas históricas (Estados, sociedad civil, individuos, gremios y otros) y, 3. la integración

30 ANCR. Serie Relaciones Exteriores. Caja nro. 164. 1905. s.f. Asamblea Iberoamericana de Paris, 1905. de la comunidad política como una idea y un programa político.

En síntesis, la región centroamericana dio entre 1902-1906 sus primeros pasos en el plano de una nueva generación de proyectos integracionistas. La constitución de una comunidad de intereses, fue progresiva $y$ no estuvo exenta de sus fracasos. Tal y como se analizó, el proceso se gestó con base en dos eventos: el Pacto de Corinto (1902) y el Tratado de Paz, Amistad y Comercio (1906). Ambos acuerdos sentaron las bases para la constitución de una comunidad política centroamericana, en la cual los Estados/Nación serán junto a otros actores de la sociedad sus protagonistas. El proceso generó tendencias discursivas sobre la integración política regional, rescató representaciones y muestras iconográficas que pusieron de relieve nuestro pasado $y$ destino común $y$, fundaron instituciones con el propósito de presentarse frente a la comunidad internacional.

\section{CITAS BIBLIOGRÁFICAS}

Acuña, Víctor Hugo. "Las concepciones de la comunidad política en Centroamérica en tiempos de la independencia (18201823)". Revista TRACE 37. 2000: 27- 40.

Astori, Danilo. "¿Hacia dónde van los procesos sub-regionales?". La reestructuración mundial y América Latina. Obstáculos en la Integración. Tomo II. México: IICEUNAM, 1994.

Balassa, Bela. Teoría de la Integración Económica. México: UTEHA, 1980.

Castañeda, Jorge. "Pan Americanism and regionalism: A mexican view". International Organization 10 (3), aug. 1956.

Cox, Robert. Production, power and word order. Columbia University Press, 1987.

Galtung, Johan. "Una teoría estructural de la integración". Revista de la integración. Economía, politica y sociología 5, noviembre. Buenos Aires: BID-INTAL: 1969. 
Hernández, Cutberto. "El Pacífico latinoamericano en los proyectos geopolíticos del siglo XIX". Geopolítica de América Latina y el Caribe. México: Instituto Panamericano de Geografía e Historia, 1999.

Keohane, Robert. After hegemony. Princenton University Press, 1984.

Mouffe, Chantal. The return of the political. London: Verso, 1993.

Pérez, Héctor. Breve historia de Centroamérica. Madrid: Alianza Editorial, 1985.

Pinto Soria, Julio César. Problemas en la formación del Estado Nacional en Centroamérica. San José: ICAP, 1983.

Pakkasvirta, Jussi. ¿Un continente una nación?. Intelectuales latinoamericanos, comunidad politica y las revistas culturales en Costa Rica y en el Perú (1919-1930). Finlandia: Academia de Ciencias y Sociedad Finlandesa de Ciencias y Letras, 1997.

Silva, Ana Margarita. "El ideario político de los intelectuales unionistas centroamericanos. 1898-1921". Ponencia VI Congreso Centroamericano de Historia. Guatemala. Diciembre, 2005.

Taracena, Arturo y Piel, Jean. Identidades nacionales y Estado moderno en Centroamérica. San José: Editorial de la Universidad de Costa Rica, 1995.

Taracena, Arturo. "Nación y república en Centroamérica. 1821-1865”. Identidades nacionales y estado moderno en Centroamérica. San José: Editorial Costa Rica, 1995. 